\title{
Reflets
}

Revue ontaroise d'intervention sociale et communautaire

\section{Les modes d'organisation du travail dans une entreprise de développement économique communautaire : le cas de la Coopérative Boréal}

Jean-Gilles Lemieux

Volume 5, numéro 1, printemps 1999

Pratiques et développement économique communautaire

URI : https://id.erudit.org/iderudit/026256ar

DOI : https://doi.org/10.7202/026256ar

Aller au sommaire du numéro

Éditeur(s)

Reflets : Revue ontaroise d'intervention sociale et communautaire

ISSN

1203-4576 (imprimé)

1712-8498 (numérique)

Découvrir la revue

Citer cet article

Lemieux, J.-G. (1999). Les modes d'organisation du travail dans une entreprise de développement économique communautaire : le cas de la Coopérative Boréal. Reflets, 5(1), 159-167. https://doi.org/10.7202/026256ar

Tous droits réservés (C) Reflets : Revue ontaroise d'intervention sociale et communautaire, 1999
Ce document est protégé par la loi sur le droit d'auteur. L'utilisation des services d'Érudit (y compris la reproduction) est assujettie à sa politique d'utilisation que vous pouvez consulter en ligne.

https://apropos.erudit.org/fr/usagers/politique-dutilisation/ 


\section{Les modes d'organisation du travail dans une entreprise de développement économique communautaire: le cas de la Coopérative Boréal ${ }^{1}$}

Jean-Gilles Lemieux

Étudiant, maîtrise en sociologie, Université Laurentienne

Le Coopérative Boréal constitue une entreprise de développement économique communautaire fort originale. Établie au sein du Collège Boréal, le premier collège d'arts et de technologie appliqués de langue française situé dans le Nord-Est de l'Ontario, la coopérative Boréal compte près de 2000 membres, 12 employés à plein temps et plus de 200 employés à temps partiel.

Fondée en 1995, un an après l'établissement du Collège, la coopérative Boréal offre de nombreux services, centrés principalement autour de la librairie et de la cafétéria. La coopérative offre plusieurs produits et services se retrouvant habituellement dans une librairie et une cafétéria : un comptoir alimentaire, un dépanneur, une billetterie d'autobus, un service de télécopie, une imprimerie et un pub. Elle offre, en plus, un service d'emplois temporaires auprès des entreprises privées et publiques, c'est-à-dire qu'elle gère un système visant à référer des apprenants et apprenantes du Collège vers des employeurs à la recherche de main d'oeuvre.

Lobjectif de départ de la Coopérative Boréal, qui était de permettre une meilleure implication des apprenants et apprenantes du Collège Boréal pendant leur séjour à l'institution de formation, 
demeure toujours primordial. Elle voulait aussi offrir aux apprenants et aux apprenantes un plus grand contrôle des dépenses consacrées aux matériaux scolaires. Cet objectif a amené les fondateurs de la coopérative à prendre en main la gestion de la cafétéria et du pub afin de pouvoir faire bénéficier les étudiants de prix réduits.

\section{Conditions favorables}

Dès ses débuts, la Coopérative a joui de l'appui de l'administration $\mathrm{du}$ Collège qui voulait créer un partenariat solide avec les apprenants et apprenantes. La Coopérative a alors bénéficié de l'implication de trois personnes convaincues du succès possible de ce projet : le directeur de la coopérative, une employée affectée au service de librairie et un employé des services alimentaires. La réussite de toute entreprise de développement économique communautaire (DÉC) exige le travail et la conviction des fondateurs ou fondatrices du projet.

À ses débuts, la Coopérative avait pour objectif de créer des emplois à temps partiel pour les apprenants et les apprenantes du Collège Boréal. Cet objectif a été atteint, il va sans dire. Qui plus est, il a aussi été nécessaire d'embaucher des employés à plein temps pour que la Coopérative fonctionne. Pour démarrer, la Coopérative a pu compter sur l'aide financière du Collège Boréal, de la Caisse populaire ainsi que de la Fédération des coopératives en milieu scolaire du Québec. Dès ses débuts, la coopérative s'est donnée une mission qui se lit comme suit: «Desservir les apprenantes et les apprenants du Collège Boréal ainsi que la collectivité francophone du Nord de l'Ontario selon les valeurs et les principes coopératifs. Ses objectifs sont au nombre de cinq:

- maintenir des prix concurrentiels dans chacun des secteurs tout en assurant la rentabilité de l'entreprise ;

- appuyer les apprenantes et les apprenants du Collège Boréal par des initiatives, entre autres, des occasions d'apprentissage et des prélèvements de fonds; 
"...une étude afin de connaître la place des employés et employées dans les initiatives d'un DÉC comme la Coopérative Boréal...»
- créer des emplois pour les apprenantes et les apprenants du Collège Boréal;

- intégrer dans ses pratiques le bien-être personnel et le respect de l'environnement;

- créer des partenariats avec d'autres organismes et coopératives susceptibles d'offrir des avantages aux apprenantes et aux apprenants ainsi qu'à la communauté.

Vu l'originalité de l'entreprise coopérative, nous avons effectué une étude afin de connaitre la place des employés et employées dans les initiatives d'un DÉC comme la Coopérative Boréal, leur niveau de participation à la vie de l'entreprise et leur niveau de satisfaction face au travail.

Pour répondre à ces questions, nous avons effectué une dizaine d'entrevues auprès des employés et employées de la Coopérative Boréal, des membres de la direction et du Conseil d'administration. Les entrevues ont porté principalement sur deux dimensions: l'organisation du travail et la dimension culturelle. Ce dernier aspect porte sur les valeurs d'intégration et d'identification des employés et employées à leur entreprise (solidarité, entraide, etc.). Par cette dimension, nous avons voulu cerner les mécanismes mis en place par la coopérative afin de susciter l'adhésion des employés et employées à la vie de la coopérative et faciliter leur intégration au fonctionnement organisationnel de l'entreprise. D'autre part, la dimension organisationnelle voulait analyser les rapports de pouvoir, le mode de gestion et les relations de travail à l'intérieur de cet organisme de développement économique communautaire.

Au niveau de l'organisation, la Coopérative est gérée par un directeur et un conseil d'administration. Le directeur est responsable des fonds et des livres comptables, du budget et de plusieurs autres fonctions déterminées par le conseil d'administration. Il est aussi responsable de la gestion du personnel. Pour sa part, le conseil d'administration est responsable des grandes questions de politiques. 


\section{La place des employés et employées dans l'organisation du travail}

Comment les employés et employées se sentent-ils face à leur travail? Sont-ils satisfaits? Sont-ils à l'aise dans leurs tâches? Dans la majorité des cas, les employés et employées se sentent très à l'aise face à leur travail. Lorsqu'ils se sentent poussés, ce n'est pas dans la gestion du travail, mais plutôt à cause de la charge de travail. De plus, les employés et employées semblent jouir d'une bonne autonomie dans l'exercice de leurs tâches et leur patron semble avoir confiance en eux. Les employés et employées se sentent confortables d'approcher le patron pour manifester leur désaccord ou pour lui faire part de ce qui ne fonctionne pas dans leur relation. Est-ce que les employés et employées participent aux décisions? Ils se sentent impliqués face aux décisions qui concernent le fonctionnement interne de la coopérative comme en témoignent les affirmations suivantes: «les patrons (soit le directeur ou le superviseur) sont des personnes très ouvertes»; «lorsqu'il y a un problème, l'honnêteté est très appréciée»; «il n'y a pas de rancune».

Cependant, lorsque les décisions relèvent de la juridiction du conseil d'administration, les employés se sentent exclus et ils n'ont pas de voix dans la prise de décision. Comme le déclare une employée:
Le Conseil d'administration n'est pas assez proche des employés et employées et les membres prennent des décisions sans savoir si la décision convient aux employés et employées. Je trouve que ce serait important d'avoir une communication avec tout le personnel, afin d'avoir les idées de tout le monde avant de dire qu'ils ont pris la décision de faire les choses de telle façon. Une entreprise qui veut s'améliorer devrait avoir cette attitude.

Quant aux relations de travail entre la direction et les employés et employées, ainsi qu'entre les employés et employées eux-mêmes, les résultats de l'enquête démontrent qu'il règne un climat de 
confiance. Pour ce qui est des rapports entre direction et employés, ces derniers sont d'avis que les relations sont très "amicales", «agréables» et «respectueuses». Le patron est ouvert aux suggestions et les employés et employées se sentent à l'aise de l'approcher. Une complicité est établie entre le patron et son personnel. Comme l'affirme une employée :

Le patron est une personne très ouverte, les relations de travail sont plaisantes parce que nous pouvons lui parler et lui donner notre opinion des choses. Le fait de travailler pour une personne agréable rend le travail et l'environnement de travail plus agréables.

En ce qui a trait aux relations entre les employés et employées, les résultats indiquent qu'elles sont très positives. Les employés et employées utilisent les termes suivants pour décrire les relations avec leurs collègues: «elle sont excellentes», «agréables», «nous formons une bonne équipe de travail», «il y a beaucoup d'entraide et de partage des tâches».

Dans toutes les entrevues, le travail d'équipe se dégage comme un élément au coeur de cette relation entre les employés et employées. La citation suivante illustre bien la position des employés et employées:

Il faut travailler en équipe. Si une ou un employé n'a pas le temps de faire quelque chose, je vais le faire ou si moi je n'ai pas le temps de faire quelque chose, il y a quelqu'un qui va m'aider. Je ne sais pas si c'est notre personnalité, mais l'entraide vient naturellement dans notre équipe de travail.

La direction a aussi souligné le travail d'équipe, l'entraide et le partage des tâches. Par exemple, le directeur général de la coopérative affirme:

À l'intérieur de la librairie, j'ai deux employées qui détiennent deux descriptions de tâches complètement différentes. Cependant, avec leur entraide et le partage des tâches de ces deux employées, je n'ai jamais été 
obligé d'organiser un horaire de travail pour faire en sorte que l'une apprenne le travail de l'autre. Cet apprentissage a été fait automatiquement et pour le bien de la coopérative. Cet exemple est aussi reproduit dans la totalité de notre entreprise. Nous avons une excellente équipe de travail.

\section{La dimension culturelle}

La dimension culturelle était aussi au coeur de cette enquête. Ainsi, le français est la langue de fonctionnement quotidien à l'intérieur de la coopérative. Les gens sont fiers d'être francophones. D'après les résultats des entrevues, les gens qui travaillent au sein de la Coopérative Boréal sont fiers de faire partie d'un collège unilingue français et de servir cette clientèle francophone. Les employés et employées ont aussi un grand sentiment d'appartenance envers leur coopérative et leur environnement de travail.

Des sources documentaires indiquent que les employés et employées de la coopérative gagnent un salaire minimal. Le faible niveau de rémunération des employés et employées de cette coopérative ne semble pas avoir nui à leur sentiment d'appartenance, aux bonnes relations de travail et aux rapports avec la clientèle. Ce qui importe pour eux, c'est le fait d'être bien dans leur travail. Comme le souligne un employé :

La seule chose que je n'aime pas de mon travail, c'est le salaire. Pour le montant de travail accompli pendant une journée, mon salaire ne suffit pas. Mais j'aime mon travail et la clientèle. 


\section{Les aspects négatifs de travailler à la Coopérative Boréal}

Les témoignages des employés et employées cités ci-haut démontrent bien les aspects positifs reliés à l'organisation du travail et à la dimension culturelle de cette entreprise. La recherche a toutefois relevé certains aspects négatifs. Le problème principal, selon les personnes interviewées c'est celui des frictions entre les employés et employées et le Conseil d'administration. D'après les employés et employées, le conseil les exclut des décisions qui les touchent. Ainsi, lorsqu'une décision est prise, il incombe à la direction de la transmettre aux employés et employées; celle-ci agit donc uniquement comme porte-parole et intermédiaire. Dans la plupart des cas, l'opinion des employés et employées est laissée pour compte. Certains employés et employées affirment que le conseil d'administration doit leur donner plus de voix dans la prise de décisions. Selon eux, lorsque les questions et les problèmes sont transmis au Conseil d'administration, le processus de prise de décision est trop long. Dans plusieurs instances, ces problèmes auraient pu tout simplement être réglés par le directeur.

Selon les employés et employées, les positions du conseil d'administration ne sont pas toujours en liens avec les valeurs et les principes coopératifs. Lorsque les dossiers sont entre les mains du Conseil d'administration, les employés et employées se sentent exclus des affaires internes de la coopérative, d'autant plus qu'ils croient pouvoir apporter un point de vue intéressant sur la prise de décisions puisqu'ils font partie du personnel de la coopérative.

Les membres du Conseil d'administration sont en général du même avis. L'un d'eux exprime l'avis que le manque d'implication des employés et employées dans la prise des décisions constitue le talon d'achille de la coopérative. D'après ce membre, la seule information donnée aux employés et employées est la décision finale transmise à la direction. Cette façon de circuler l'information pose problème parce que la direction détient seulement un vote au Conseil d'administration et ne peut pas représenter l'ensemble 
des employés et employées. À cette difficulté s'ajoute le fait qu'il n'y a pas de bulletin d'information, de journal ou de rapport pour renseigner les employés et employées sur la prise de décisions et sur le contenu des réunions. À l'heure actuelle, le conseil n'envisage pas de régler ou de corriger cette situation.

\section{Conclusion}

Tout indique que dans la Coopérative Boréal, les employés et employées sont impliqués dans la vie de l'entreprise et semblent satisfaits de leur travail. Cependant, il est important de faire une différence entre la gestion quotidienne et la gestion globale de la coopérative. Dans la gestion quotidienne de l'entreprise, les employés et employées prennent des décisions, aiment leur travail et déclarent avoir plus d'autonomie dans l'exercice de leur tâches. De plus, ils ne sont pas perçus uniquement comme des salariés, mais aussi comme des francophones, porteurs de langue et de culture. La direction et les employés et employées de la Coopérative Boréal se disent fiers d'être francophones, d'accorder une grande importance au travail d'équipe, aux relations de travail et aux relations avec la clientèle. Pour eux, le fait d'appartenir à cette équipe de travail, de se sentir utiles et essentiels au bon fonctionnement de l'entreprise, démontre qu'être de culture francophone est une valeur dominante au sein de la coopérative.

Les résultats de cette enquête ne contredisent pas la littérature qui défend le rôle positif que joue le DÉC, mais nuancent quelque peu l'optimisme que l'on y retrouve. Il me semble que les coopératives demeurent une forme de travail distincte de celui des entreprises privées; néanmoins, les coopératives sont des institutions politiques, c'est-à-dire, traversées par des conflits, des rapports de force et c'est cet aspect que sous-estime la littérature. Quoiqu'elles soient des entreprises particulières, les coopérations ne peuvent échapper aux rapports de force qui caractérisent la dynamique des rapports sociaux dans une organisation de travail. 


\section{Note}

1. Lemieux, J.G., (1998) (s. la dir. de Rachid Bagaoui et Donald Dennie), Succès et limites des modes d'organisation du travail dans les entreprises du développement économique communautaire: le cas de la Coopérative Boréal limitée, Mémoire de spécialisation, Département de sociologie, Université Laurentienne. 